\title{
ARTICLE
}

Received 28 Sep 2015 | Accepted 19 Apr 2016 | Published 25 May 2016

\section{Plastic anisotropy and dislocation trajectory in BCC metals}

Lucile Dezerald $^{1,2}$, David Rodney ${ }^{3}$, Emmanuel Clouet ${ }^{1}$, Lisa Ventelon ${ }^{1} \&$ François Willaime ${ }^{4}$

Plasticity in body-centred cubic (BCC) metals at low temperatures is atypical, marked in particular by an anisotropic elastic limit in clear violation of the famous Schmid law applicable to most other metals. This effect is known to originate from the behaviour of the screw dislocations; however, the underlying physics has so far remained insufficiently understood to predict plastic anisotropy without adjustable parameters. Here we show that deviations from the Schmid law can be quantified from the deviations of the screw dislocation trajectory away from a straight path between equilibrium configurations, a consequence of the asymmetrical and metal-dependent potential energy landscape of the dislocation. We propose a modified parameter-free Schmid law, based on a projection of the applied stress on the curved trajectory, which compares well with experimental variations and first-principles calculations of the dislocation Peierls stress as a function of crystal orientation.

\footnotetext{
${ }^{1}$ DEN-Service de Recherches de Métallurgie Physique, CEA, Université Paris-Saclay, F-91191 Gif-sur-Yvette, France. ${ }^{2}$ Institut Jean Lamour, Université de Lorraine - CNRS, F-54011 Nancy, France. ${ }^{3}$ Institut Lumière Matière, Université Lyon 1 - CNRS, F-69622 Villeurbanne, France. ${ }^{4}$ DEN-Département des Matériaux pour le Nucléaire, CEA, Université Paris-Saclay, F-91191 Gif-sur-Yvette, France. Correspondence and requests for materials should be addressed to L.D. (email: lucile.dezerald@univ-lorraine.fr).
} 
T he crystalline structure of metals strongly affects their plasticity. In face-centred cubic and hexagonal closepacked metals with basal slip, plastic flow sets in when the resolved shear stress (RSS, the component of the applied stress tensor that produces a shear stress along the slip direction and parallel to the slip plane) reaches a critical value. In this yield criterion, famously known as the Schmid law ${ }^{1}$, the critical RSS is a structural parameter dependent on the thermomechanical history of the metal, but not on the sense nor on the direction of the applied stress.

Body-centred cubic (BCC) metals are known to disobey the Schmid law, in particular because the critical RSS depends on the orientation and sense of the applied stress ${ }^{2-4}$. BCC metals slip along dense $\langle 111\rangle$ directions, but the potential slip planes vary depending on the element, the temperature and orientation of the maximum RSS plane ${ }^{3,5}$. Plastic anisotropy in these metals was initially interpreted as resulting from slip on $\{112\}$ planes $^{2,6}$. The reason is that these planes are not symmetrical with respect to shear along $\langle 111\rangle$, with a direction leading to twinning $(\mathrm{T})$, softer than the opposite, antitwinning (AT), direction. However, this hypothesis does not hold, as the T/AT asymmetry remains at low temperatures in $\alpha$-Fe and in metals from group VI (Mo and W), which slip along $\{110\}$ planes that are symmetrical with respect to $\langle 111\rangle$ shear $^{3,7}$. Moreover, screw dislocations with $1 / 2\langle 111\rangle$ Burgers vectors, which are known to be the source of the plastic anisotropy ${ }^{4,8}$, have been shown via atomistic calculations to disobey the Schmid law even when they glide on $\{110\}$ planes ${ }^{9-11}$. Another feature, perhaps less commonly known, is that deviations from the Schmid law are metal dependent ${ }^{12}$.

To describe non-Schmid effects, a phenomenological yield criterion was proposed as a linear combination of the stresses that affect plasticity ${ }^{13,14}$. The stresses were identified through atomistic simulations $s^{9,14,15}$ and the resulting generalized yield criterion was shown to reproduce accurately atomistically calculated anisotropic Peierls stresses, that is, the critical RSS to set an isolated screw dislocation in motion in the absence of thermal activation ${ }^{10,14,16,17}$. However, there has been so far no physical explanation of the dependence of the yield criterion on these various stress components nor of the variation among metals of the parameters entering the law, reflecting our lack of understanding of the T/AT asymmetry.

We show here using density functional theory (DFT) calculations that the link between the T/AT asymmetry and screw dislocations lies in the dislocation trajectory that deviates from the average $\{110\}$ slip plane. Applying the Schmid law to the actual dislocation trajectory instead of the average $\{110\}$ glide plane allows the physical interpretation of the phenomenological generalized yield criterion and leads to predictions of T/AT asymmetries that are in qualitative agreement with experiments and in quantitative agreement with DFT calculations of the Peierls stress.

\section{Results}

T/AT asymmetry. To examine plastic anisotropy, we use firstprinciples DFT calculations to determine the energetics and trajectories of $\mathbf{b}=1 / 2[111]$ screw dislocations gliding between neighbouring equilibrium configurations. We consider the $0 \mathrm{~K}$ Peierls stress limit where dislocations glide rigidly, allowing to simulate straight periodic dislocation lines. The calculations are performed both in the absence and in the presence of a shear stress applied in the maximum RSS plane. This plane belongs to the [111] zone but may differ from the $(1 \overline{1} 0)$ plane, as encountered in tensile tests (Fig. 1a). We considered the following BCC transition metals: $\mathrm{V}, \mathrm{Nb}$ and $\mathrm{Ta}$ from group $\mathrm{V}$; Mo and $\mathrm{W}$ from group VI; and Fe. The energy barriers against glide, as a function of the applied stress and orientation of the maximum RSS plane, are obtained by a constrained minimization and the dislocation positions defining the corresponding trajectory are deduced from the stress variations calculated along the paths (for details, see Methods).

Figure $1 \mathrm{~b}$ shows the BCC structure projected in the (111) plane, along with the high-symmetry positions of the $1 / 2\langle 111\rangle$ screw dislocation. The easy core configuration is centred on upward triangles formed by three [111] atomic columns and corresponds to the minimum-energy stable position of the dislocation. The hard core is an unstable position centred on downward triangles, where the three [111] columns are at the same altitude. We used the split core configuration to model the dislocation in the vicinity of a [111] atomic column. It corresponds to altitude shifts of $\pm b / 6$ for the two opposing columns (columns A and B for a split core near column $\mathrm{S}$ in the ABS triangle $)^{18,19}$. The split core is unstable in DFT calculations 99,20 but metastable with some interatomic potentials $^{21-23}$. The T/AT asymmetry arises when a simple shear stress is applied along the [111] direction with a maximum
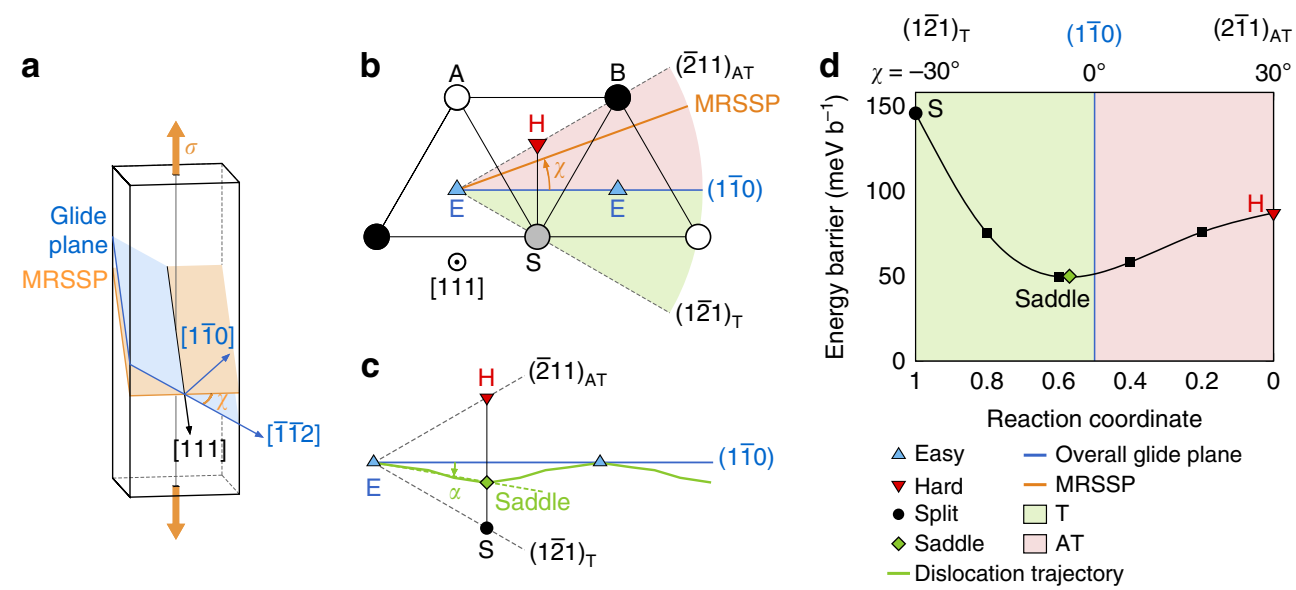

Figure 1 | BCC metals plasticity and crystallography. (a) Schematic representation of the (110) glide plane and maximum RSS plane (MRSSP) during a tensile test. (b) Projection of the BCC lattice in the (111) plane showing the high-symmetry dislocation positions (E, easy core; $\mathrm{H}$, hard core; $\mathrm{S}$, split core). The angle $\chi$ between the (110) plane and the maximum RSS plane is positive in the AT region (red area) and negative in the T region (green area). (c) Example of dislocation trajectory (green line) calculated with DFT in Mo and definition of the deviation angle $\alpha$. The green diamond shows the saddle point position. (d) Energy profile along the hard-split line (H-S segment in $\mathbf{b}, \mathbf{c}$ ) calculated with DFT in Mo. 
RSS plane that is not the central (110) plane $e^{4}$. The orientation of the maximum RSS plane is commonly referred to by its angle $\chi$ relative to the (110) plane, as illustrated in Fig. 1a,b. $\{112\}$ planes are not symmetrical when sheared along the [111] direction. For positive stresses, when $\chi=+30^{\circ}$, the (211) plane is sheared in the AT direction, whereas when $\chi=-30^{\circ}$, the $(1 \overline{2} 1)$ plane is sheared in the $\mathrm{T}$ direction. The region with $\chi>0$ (respectively $\chi<0)$ is therefore referred to as antiwinning (respectively twinning). Interestingly, the T/AT asymmetry is also visible in the topology of the (111) plane (see Fig. 1b), as the ( $\overline{2} 11)$ plane in the AT region contains the hard core, whereas the $(1 \overline{2} 1)$ plane in the $\mathrm{T}$ region contains a [111] atomic column. Therefore, a shear stress applied along a maximum RSS plane with $\chi>0$ pushes the dislocation towards the hard core, whereas when $\chi<0$ the dislocation is pushed towards the split core.

Dislocation trajectory. The Peierls barriers between two neighbouring easy cores were calculated in all BCC metals and the corresponding dislocation trajectories were determined from the stress variations observed along the paths (see Methods). Extracting these trajectories from the atomistic simulations through a fit of the atomic displacements rather than using the stress variation leads qualitatively to the same trajectories (see Supplementary Fig. 1). An example of dislocation trajectory in the absence of an applied stress is shown in Fig. 1c. As expected from previous studies ${ }^{9,20,24-27}$, the trajectory is not straight and the maximum deviation is reached halfway across the path when the dislocation is in the saddle state of maximum energy between

\section{Table 1 | Deviation angle and energy ratios from DFT calculations ${ }^{20}$ in the absence of applied stress in all BCC} transition metals.

\begin{tabular}{lcccccc} 
& $\mathbf{v}$ & $\mathbf{N b}$ & $\mathbf{T a}$ & $\mathbf{M o}$ & $\mathbf{W}$ & $\mathbf{F e}$ \\
\hline$\alpha\left(^{\circ}\right)$ & -10.6 & -21.7 & -7.1 & -9.9 & -10.9 & -1.7 \\
$E_{\mathrm{S}} / E_{\mathrm{H}}$ & 0.98 & 1.03 & 1.48 & 1.67 & 1.62 & 2.73
\end{tabular}

$\mathrm{BCC}$, body-centred cubic; DFT, density functional theory. a

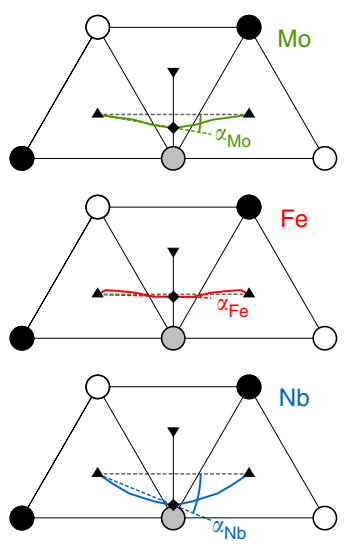

b

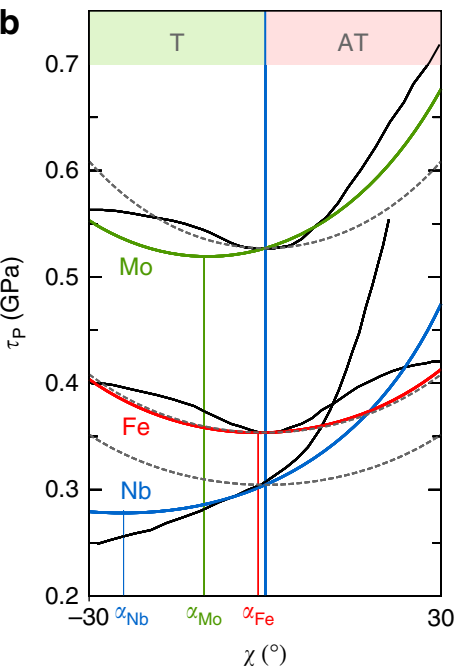

Figure 2 | Dislocation trajectory and Schmid law deviation in BCC metals. (a) Dislocation trajectory between easy core configurations obtained by DFT in $\mathrm{Nb}, \mathrm{Mo}$ and $\mathrm{Fe}$, with the corresponding deviation angle $\alpha$. (b) Peierls stress variations with crystal orientations predicted by the modified Schmid law of equation (2) (coloured lines) and compared with the experimental data at $4.2 \mathrm{~K}$ (ref. 12) (black lines). Schmid law is indicated in dashed grey lines. the initial and final configurations. At this point, the dislocation trajectory crosses the line that connects the hard core to the split core configurations, hereafter called the hard-split line. As the trajectories are close to piecewise linear, we will characterize the deviation of a path from the straight line by the angle $\alpha$ between the first section of the trajectory and the horizontal (110) plane (see Fig. 1c).

The $\alpha$ angles computed in the absence of applied stress are given in Table 1 . They are systematically negative, reflecting that the deviation always points towards the [111] atomic column located in the $\mathrm{T}$ region. This can be understood from the dislocation two-dimensional (2D) Peierls potential (the energy landscape that describes the interaction between the dislocation and the crystal lattice) $)^{20,24-28}$. The energy along the hard-split line calculated with DFT is illustrated in Mo in Fig. 1d. This energy profile is strongly asymmetric with respect to the central (11i0) plane and the local minimum corresponding to the saddle point of the 2D Peierls potential is shifted towards the split core in the $\mathrm{T}$ region. As the gliding dislocation tends to follow the path of minimum energy on the Peierls potential, the shift of the

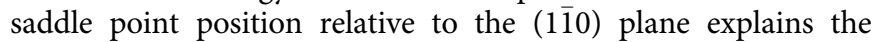
negative $\alpha$ angles in Table 1 .

We also note that the magnitude of the deviation of the dislocation trajectory is strongly metal dependent. As illustrated in Fig. 2a, the deviation is largest in $\mathrm{Nb}$, where the dislocation passes close to the [111] column, intermediate in Mo and minimum in $\alpha-\mathrm{Fe}$, where the path is almost straight. This is a direct consequence of the asymmetry of the hard-split line, which is itself strongly metal dependent ${ }^{20}$. We reported in Table 1 the energy ratios between the split and hard cores, $E_{\mathrm{S}} / E_{\mathrm{H}}$, taking as a reference the easy core. We see that, with the exception of $\mathrm{V}$, the split core energy relative to the hard core energy controls the saddle point position, that is, the larger the $E_{S} / E_{\mathrm{H}}$ ratio, the smaller the deviation angle. In particular, $\mathrm{Fe}$ has the largest ratio and the lowest $\alpha$-value, whereas $\mathrm{Nb}$ has an $E_{\mathrm{S}} / E_{\mathrm{H}}$ ratio close to 1 and the largest deviation angle. The reason why the energy ratios are metal dependent remains to be understood; however, further explanations require investigations that are out of the scope of the present publication.

Modified Schmid law. The Schmid law assumes that the critical RSS is controlled by the shear stress projected on the glide plane. If a shear stress $\tau$ is applied in the maximum RSS plane, the RSS in the (11 0$)$ glide plane is $\tau \cos (\chi)$. Applying the Schmid law, the screw dislocation Peierls stress should then vary with the maximum RSS plane orientation as:

$$
\tau_{\mathrm{P}}(\chi)=\frac{\tau_{\mathrm{P}}^{0}}{\cos (\chi)},
$$

which is symmetrical with respect to the central (110) plane where $\chi=0^{\circ}$. However, we have shown above that in BCC metals, the dislocation trajectory is (110) only on average. To obtain the Peierls stress, one should therefore project the RSS not on the average (110) plane but rather on the actual trajectory. As the trajectories are close to piecewise linear with the unstable position of the dislocation under stress in the first half of the path, we propose to project the RSS on the plane inclined by an angle $\alpha$ with respect to the horizontal (110) plane. We thus obtain a modified Schmid law, expressed as:

$$
\tau_{\mathrm{P}}(\chi)=\frac{\tau_{\mathrm{P}}^{*}}{\cos (\chi-\alpha)},
$$

depending on two material-dependent parameters, $\tau_{\mathrm{P}}^{*}$ and $\alpha$. 
Comparison with experiments. To test the accuracy of the above law, we wish to compare its predictions with experimental data. We will consider three metals, $\mathrm{Nb}, \mathrm{Mo}$ and $\mathrm{Fe}$, for which very low-temperature $(4.2 \mathrm{~K})$ measurements of the yield stress variations with crystal orientation are available and provide the best experimental estimates for the screw dislocation Peierls stress ${ }^{12}$. Reproducing Peierls stresses from atomistic calculations is notoriously difficult ${ }^{29-31}$ because of the need to account for zero-point vibrations that play an important role in dislocation glide at low temperatures ${ }^{31,32}$ and non-glide stresses that strongly affect the Peierls stress ${ }^{14,16,17,33}$. Both effects would be extremely computationally expensive to include. For this reason, and because we are interested here in the asymmetry of the Peierls stress rather than its magnitude, we have taken the values of the $\alpha$-angles from our DFT zero-stress calculations in Table 1; however, we have chosen $\tau_{\mathrm{P}}^{*}$ so as to reproduce the experimental value at $\chi=0^{\circ}$.

The predictions from equation (2) are compared with the experimental data in Fig. 2b. We obtain a qualitatively good agreement, especially considering the simplicity of equation (2). It is noteworthy that we recover that $\mathrm{Nb}$ has the strongest T/AT asymmetry, Mo is intermediate and $\alpha$-Fe is almost symmetrical. This agreement shows that the T/AT asymmetry is a direct consequence of the deviation of the dislocation trajectory from a straight path, with larger deviations (more negative $\alpha$-angles, or equivalently, saddle states closer to a [111] atomic column) leading to larger asymmetries. The fact that the yield stress is systematically lower in the $\mathrm{T}$ region than in the AT region ${ }^{3}$ is therefore directly connected to $\alpha$ being negative in all BCC metals. Discrepancies between the modified Schmid law and the experiments may arise from non-glide stresses that are present in the experiments but are not accounted for in equation (2).

So far, non-Schmid effects have been modelled using a phenomenological law based on a linear combination of the stresses that affect dislocation motion, which in the case of the T/AT asymmetry are the RSSs projected on two different planes of the $\langle 111\rangle$ zone $^{14}$. The choice of these planes is arbitrary as long as they are not parallel ${ }^{33,34}$. When the horizontal $(1 \overline{1} 0)$ plane and the (101) plane in the twinning region are chosen, the yield criterion is expressed as:

$$
\tau_{\mathrm{P}}(\chi)=\frac{\tau_{\mathrm{P}}^{1}}{\cos (\chi)+a \cdot \cos (\chi+\pi / 3)},
$$

with $\tau_{\mathrm{p}}^{1}$ and $a$, two phenomenological parameters that may be fitted on experimental or numerical data. Expanding and reorganizing equations (2) and (3), we can show that $\tau_{\mathrm{P}}^{1}=\sqrt{3} \tau_{\mathrm{P}}^{*} /[2 \cos (\pi / 6-\alpha)]$ and $\mathrm{a}=-\sin (\alpha) / \cos (\pi / 6-\alpha)$. Equation (2) therefore provides a physical justification for the phenomenological law in equation (3). In particular, the relation above shows that $a$ increases monotonically from 0 to 1 when $\alpha$ decreases from 0 (straight path) to $-\pi / 6$ (path passing through the [111] atomic column). The parameter $a$ is therefore directly related to the deviation of the dislocation trajectory from the straight $(1 \overline{1} 0)$ plane.

DFT calculations under stress. To show that a quantitative agreement can be obtained with the modified Schmid law and avoid the difficulties mentioned above related to the comparison between experimental and atomistic data, we will now compare the predictions from equation (2) with DFT calculations of the Peierls stress. We consider metals from group VI, Mo and W, which slip along the $\{110\}$ plane of maximum RSS at low temperatures $^{12}$. Given the computational load related to such calculations, we studied three orientations: $\chi=0^{\circ}, \chi=-20^{\circ}$ in the $\mathrm{T}$ region and $\chi=+20^{\circ}$ in the AT region.

To obtain Peierls stresses, we did not compute maximum derivatives on 2D Peierls potentials that are not accurate enough, but directly determined the applied stress that cancels the Peierls barrier. We first determined Peierls barriers under increasing simple shear stresses, $\tau$, applied along the three orientations just mentioned (see Methods). To reduce the computational load, we computed only the first half of the paths where the activated state is located. The results are illustrated in Fig. 3 for Mo. As expected, the enthalpy barriers decrease with increasing applied stress. The corresponding dislocation trajectories do not depend on the applied stress, except for a small offset due to the displacement of the dislocation equilibrium position from the bottom of the

a
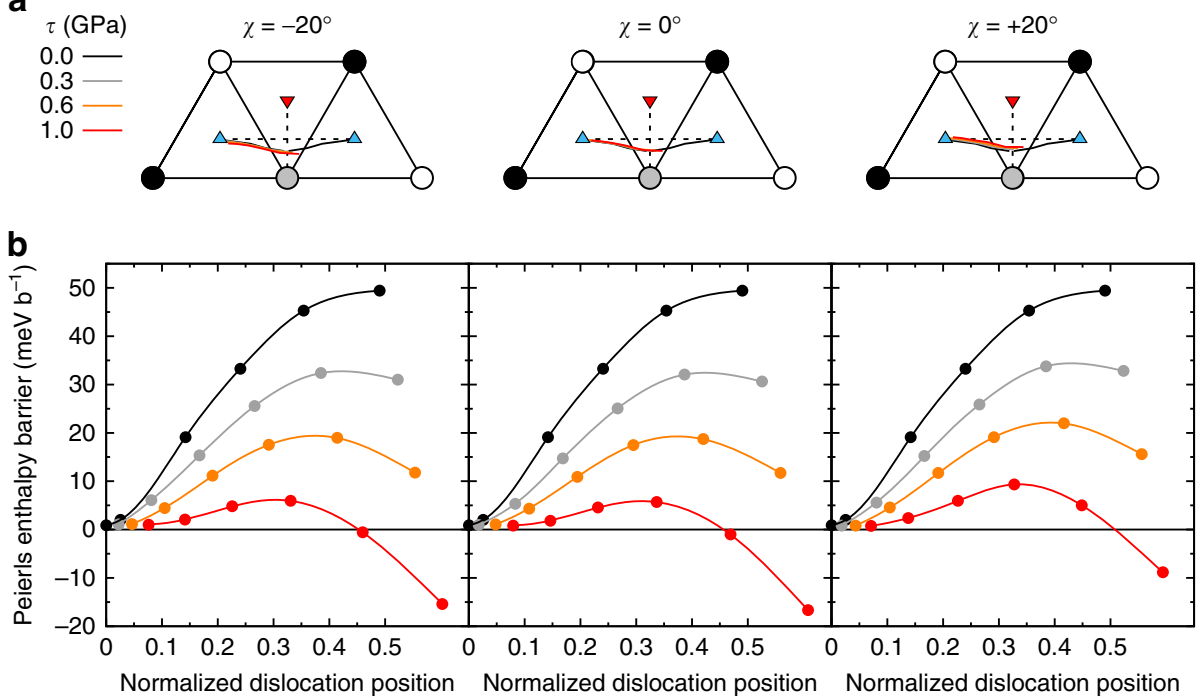

Figure 3 | Dislocation trajectories and Peierls barriers under stress in Mo. Only the first half of the paths, which contain the enthalpy maxima, was calculated by DFT. In $\mathbf{a}$, in the absence of applied stress, full trajectories are shown, owing to their symmetry (black lines). In $\mathbf{b}$, dislocation positions are projected on the horizontal (110) plane and normalized between 0 and 1 when the dislocation moves between the initial and final easy core configurations. 


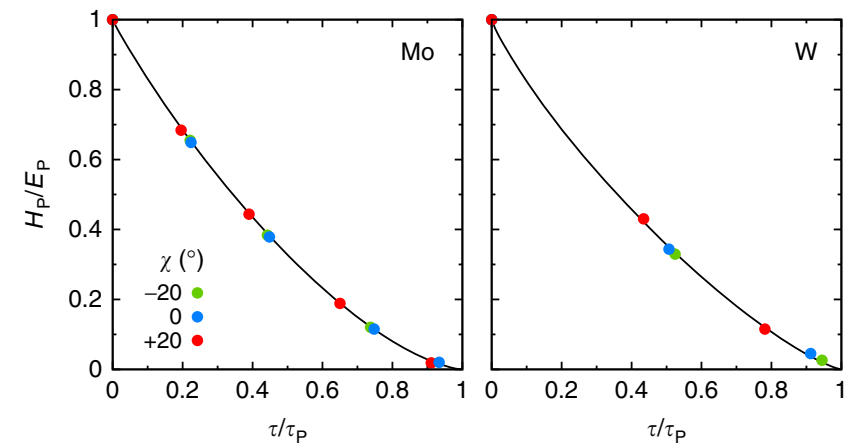

Figure 4 | Normalized Peierls enthalpy variations with applied stress in Mo and $\mathbf{W}$. The Peierls enthalpy $H_{\mathrm{P}}$ is normalized by the energy barrier, $E_{\mathrm{P}}$ calculated with DFT at zero applied stress. The applied stress, $\tau$, is normalized by the Peierls stress, $\tau_{\mathrm{P}}$ for each orientation. The symbols are the DFT data, the black lines the fits by Kocks-type power laws. We found that a single set of $p$ and $q$ parameters fitted all data for a given material $((p, q)=(0.94,1.51)$ for $M o$ and $(0.85,1.27)$ for $W)$

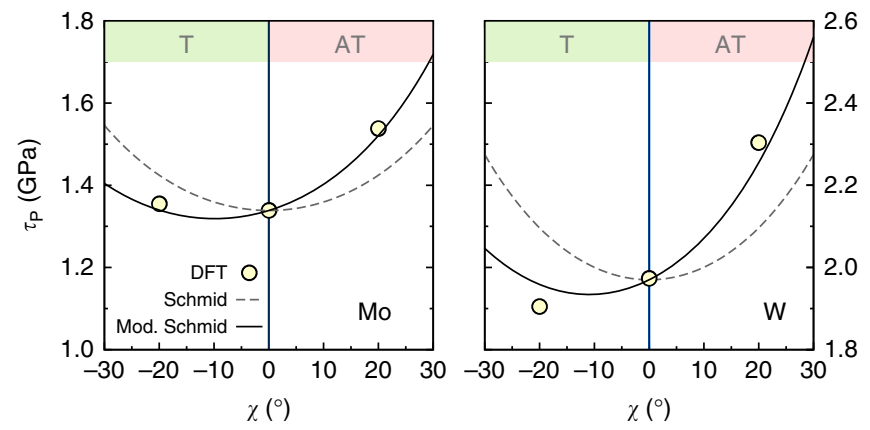

Figure 5 | Peierls stress variations with crystal orientation in Mo and $\mathbf{W}$.

The Peierls stress variations calculated with DFT under stress (circles) are compared with the original Schmid law (dashed lines) and with the modified Schmid law from equation (2) (full lines).

Peierls valley. This will allow us in the following to use the values of $\alpha$ computed in the absence of applied stress (Table 1) to parametrize equation (2). From the barriers calculated at different applied stresses and orientations, we extracted the Peierls enthalpies, $H_{\mathrm{P}}(\tau, \chi)$, the maximum of the Peierls barriers. The Peierls stresses, $\tau_{\mathrm{P}}(\chi)$, were then obtained as the critical stresses at which the Peierls enthalpies disappeared. For this, we used a power law fit of Kocks type ${ }^{35}, H_{\mathrm{P}}(\tau, \chi)=E_{\mathrm{P}}\left[1-\left(\tau / \tau_{\mathrm{P}}(\chi)\right)^{p}\right]^{q}$. This law was originally proposed to model phenomenologically the stress dependence of the kink-pair formation enthalpy but, interestingly, we found here that the Peierls enthalpies follow the same trend. Our fits are therefore not to be used to predict dislocation kinetics, but only to extract Peierls stresses, $\tau_{\mathrm{P}}(\chi)$. Moreover, as shown in Fig. 4, we performed calculations near the fitted $\tau_{\mathrm{P}}(\chi)$, to ensure that the Peierls enthalpies vanished at these stresses.

From the results shown in Fig. 4, we obtained the Peierls stress variations with maximum RSS plane orientation in Mo and $\mathrm{W}$ displayed in Fig. 5 (circles). We find that $\mathrm{W}$ is more asymmetric than Mo, which is consistent with the fact that the $\alpha$-angle in $\mathrm{W}$ is more negative than in Mo (see Table 1). In Fig. 5, we also reported the Peierls stress variations predicted by equation (2) (solid line). The values of $\tau_{\mathrm{p}}^{*}$ in this equation were set to reproduce the Peierls stresses obtained by DFT at $\chi=0^{\circ}$. We see that there is a very good agreement between the values obtained with DFT and the Peierls stresses predicted by the modified
Schmid law. This confirms the accuracy of this law and the fact that the T/AT asymmetry is intimately related to the deviation of the dislocation trajectory from a straight line between equilibrium configurations.

Obviously, the present law is not limited to DFT calculations and can be applied to interatomic potentials. In particular, we know that the embedded atom method (EAM) potential developed by Mendelev et al. ${ }^{23}$ for $\mathrm{Fe}$ predicts a doublehumped Peierls barrier that passes through the split core configuration, which is metastable with this potential ${ }^{19-22,28}$. As a result, we know without calculation that $\alpha=-\pi / 6$. In this case, equation (2) predicts the strongest T/AT asymmetry. This is confirmed by the Peierls stress calculations presented in ref. 10 and compared with equation (2) in Supplementary Fig. 2.

\section{Discussion}

We showed that plastic anisotropy is linked to the $1 / 2\langle 111\rangle$ screw dislocation trajectory and, more specifically, to its deviation from the $(1 \overline{1} 0)$ average glide plane. The deviation is itself related to the position of the saddle configuration systematically located in the $\mathrm{T}$ region. As the dislocation $2 \mathrm{D}$ Peierls potential is metal dependent, so is the deviation and thus the T/AT asymmetry. This origin of plastic anisotropy, evidenced by atomistic simulations, can be accounted for in a modified version of the Schmid law, where we showed that the component of the applied stress tensor that affects dislocation motion is the shear stress resolved on the actual inclined trajectory rather than the average (110) plane. The saddle point located in the $\mathrm{T}$ region thus reconciles the apparent paradox of a core energy higher for the split than for the hard core with simultaneously a resistance lower in the T region than in the AT region ${ }^{19,20,28}$.

With this new understanding of the T/AT asymmetry at $0 \mathrm{~K}$, it will now be possible to model plastic anisotropy at finite temperatures. Line tension models, using the Peierls barrier as input parameter, have proven to faithfully describe the kink-pair mechanism operating at finite temperatures down to rather low stresses $^{26,36,37}$. To investigate the T/AT asymmetry evolution with temperature, 2D Peierls potentials will need to be considered. Using phenomenological potentials, Edagawa et al. ${ }^{24,25}$ have shown that this approach reproduces the temperature dependence of the T/AT asymmetry observed experimentally in BCC transition metals. One should now employ atomistically determined 2D potentials ${ }^{20}$. We expect that the dislocation segments inside the nucleating kink pairs will follow a curved path similar to the straight dislocation, to minimize their core energy, confirming the importance of the dislocation trajectory on the T/AT asymmetry at finite temperatures.

It will also now be of prime importance to consider the other aspect of plastic anisotropy, that is, the effect of non-glide stresses. These stresses are resolved perpendicularly to the dislocation Burgers vector. They do not produce any force on the dislocation but still strongly affect its core structure, Peierls stress and mobility ${ }^{9,15}$. Phenomenologically, non-glide stresses have been incorporated in the linear combination of equation (3) (refs 16,17,38), but it remains to be evaluated how these stresses affect the dislocation trajectory and whether they may be incorporated in equation (2) as an additional dependence of the deviation angle. Such DFT calculations are extremely computationally expensive but would allow for a complete and physical picture of plastic anisotropy in BCC metals.

\section{Methods}

DFT calculations. The first-principles electronic structure calculations were performed within the DFT framework using the PWSCF plane-wave code ${ }^{39}$. The pseudopotentials are ultrasoft with semicore electrons for group V and VI 
elements, and without semicore electrons but with nonlinear core corrections for Fe. Residual forces after relaxation are smaller than $10^{-2} \mathrm{eV} \AA^{-1}$. The HermiteGauss scheme to broaden the electronic density of states was used with a smearing of $0.3 \mathrm{eV}$. The calculations in Fe are spin polarized and the Perdew-BurkeErnzerhof generalized gradient approximation was used with a wave-function cutoff of 40 Ry and a shifted $1 \times 2 \times 16 k$-point grid for all elements. No symmetry operator was applied apart from the inversion symmetry. The calculations were performed using a periodic array of dislocation dipoles with a quadrupolar arrangement, as in previous studies ${ }^{19,20,40,41}$, where we have carefully checked that we correctly obtain the properties of an isolated dislocation by correcting for the elastic interactions with the periodic images. In particular, we showed that the position of the saddle point does not depend on the cell size (see Fig. 15 in ref. 20).

Calculations under stress. We applied pure shear stresses along the [111] axis:

$$
\sigma=\tau\left(\begin{array}{ccc}
0 & 0 & -\sin (\chi) \\
0 & 0 & \cos (\chi) \\
-\sin (\chi) & \cos (\chi) & 0
\end{array}\right),
$$

where $\tau$ is the stress resolved in the maximum RSS plane and $\chi$ is the angle between the maximum RSS plane and the (11̄0) plane (see Fig. 1). Stresses were applied by straining the periodicity vectors of the simulation cell, with the applied strain $\varepsilon$ related to the target stress by ${ }^{41}$ :

$$
\sigma_{i j}=C_{i j k l}\left(\varepsilon_{k l}+\frac{b_{k} A_{l}+b_{l} A_{k}}{2 S}\right),
$$

where $S$ is the area of the simulation cell perpendicular to the dislocation lines and $C_{i j k l}$ are the elastic constants computed from ab initio. For dislocations with a line direction along the $z$ axis, the cut vector $\vec{A}$ is given by $A_{x}=y_{1}-y_{2}$ and $A_{y}=x_{2}-x_{1}$, where $\left(x_{1}, y_{1}\right)$ and $\left(x_{2}, y_{2}\right)$ are the coordinates of the dislocations with Burgers vectors $\vec{b}$ and $-\vec{b}$, respectively.

Peierls barriers. The Peierls barriers were calculated at fixed periodicity vectors by displacing one dislocation of the dipole, while the second dislocation remained immobile (see Supplementary Fig. 3). The initial paths were discretized linearly and the first half of each path was relaxed using the reaction coordinate method ${ }^{42}$, which is known to predict accurately minimum energy paths in the case of simple paths as considered here ${ }^{19}$. To avoid the reaction coordinate being maintained by the addition of small displacements spread over all the atoms of the simulation cell (instead of large displacements around the dislocation core as intended), the constraint was imposed only to the five atoms closest to the dislocation core. As the distance between dislocations varies along the paths, so does their elastic interaction energy. Once the dislocation positions were determined (see below), we withdrew this elastic contribution from the $a b$ initio energy variation using anisotropic linear elasticity ${ }^{20,41,43}$ (see Supplementary Fig. 3 for a validation of this approach).

Dislocation position. Because of the fixed periodicity vectors and of the varying distance between the two dislocations composing the dipole, a stress variation is observed in the simulations. One can deduce from this variation the dislocation positions $^{44}$. Inverting the system in equation (5) for the $\langle 111\rangle$ screw dislocation, the positions are given by:

$$
\begin{gathered}
x_{1}-x_{2}=-\frac{S}{b}\left[\frac{C_{66} \sigma_{y z}+C_{15} \sigma_{x y}}{C_{44} C_{66}-C_{15}^{2}}-2 \varepsilon_{y z}\right], \\
y_{1}-y_{2}=\frac{S}{b}\left[\frac{\left(C_{11}-C_{12}\right) \sigma_{x z}-C_{15}\left(\sigma_{x x}-\sigma_{y y}\right)}{\left(C_{11}-C_{12}\right) C_{44}-2 C_{15}^{2}}-2 \varepsilon_{x z}\right],
\end{gathered}
$$

with the elastic constants written in Voigt notation in the dislocation frame of

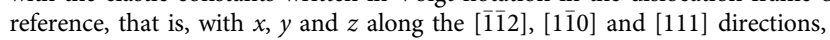
respectively. Equations (6) and (7) provide the relative positions of the dislocations. To obtain absolute positions, we first consider that, when dislocations are at rest, that is, in their equilibrium initial and final positions, they are both displaced by the same distance from the bottom of their Peierls valleys but in opposite directions. We then assume that the position of the dislocation staying in the same Peierls valley varies linearly between the initial and final configurations, a reasonable assumption in view of the small displacement experienced by this dislocation compared with the gliding dislocation.

We checked that the predictions of the modified Schmid law do not rely on the definition used for the dislocation position by performing all the postprocessing mentionned above with a second definition for the dislocation position: the 'cost function method' that consists in adjusting the atomic positions calculated with DFT to the atomic positions calculated for a compact screw dislocation within anisotropic elasticity by minimization of a cost function. This method is detailed in refs 19,20. All the obtained data are given in Supplementary Figs 1 and 4 and in Supplementary Table 1 . The values of $\alpha$ calculated with the cost function method follows the same trend as those calculated with the stress-based method and we find again a very good agreement between the DFT values and the predictions from the modified Schmid law, thus confirming the validity of the present approach.
Data availability. The data that support the findings of this study are available from the corresponding author on request.

\section{References}

1. Schmid, E. in Proceedings of the First International Congress of Applied Mechanics (eds Biezeno, C. \& Bergers, J.) 342-353 (Delft, 1924).

2. Taylor, G. I. The deformation of crystals of $\beta$-brass. Proc. R. Soc. Lond. A 118, 1-24 (1928).

3. Christian, J. W. Some surprising features of the plastic deformation of bodycentered cubic metals and alloys. Metall. Trans. A 14, 1237-1256 (1983).

4. Duesbery, M. S. \& Vitek, V. Plastic anisotropy in bcc transition metals. Acta Mater. 46, 1481-1492 (1998).

5. Taylor, G. I. \& Elam, C. F. The distortion of iron crystals. Proc. R. Soc. Lond. A 112, 337-361 (1926)

6. Pichl, W. Slip geometry and plastic anisotropy of body-centered cubic metals. Phys. Stat. Sol. (a) 189, 5-25 (2002).

7. Caillard, D. Kinetics of dislocations in pure Fe. Part II. In situ straining experiments at low temperature. Acta Mater. 58, 3504-3515 (2010).

8. Kubin, L. P. The low-temperature mechanical properties of B. C. C. metals and their alloys. Rev. Deform. Behav. Mater. 4, 181-275 (1982).

9. Ito, K. \& Vitek, V. Atomistic study of non-Schmid effects in the plastic yielding of bcc metals. Phil. Mag. A 81, 1387-1407 (2001).

10. Chaussidon, J., Fivel, M. \& Rodney, D. The glide of screw dislocations in BCC Fe: atomistic static and dynamic simulations. Acta Mater. 54, 3407-3416 (2006).

11. Gröger, R., Bailey, A. G. \& Vitek, V. Multiscale modeling of plastic deformation of molybdenum and tungsten: I. atomistic studies of the core structure and glide of $1 / 2\langle 111\rangle$ screw dislocations at $0 \mathrm{~K}$. Acta Mater. 56, 5401-5411 (2008).

12. Aono, Y., Kuramoto, E. \& Kitajima, K. Orientation dependence of slip in niobium single crystals at 4.2 and 77 K. Scripta Mater. 18, 201-205 (1984).

13. Qin, Q. \& Bassani, J. L. Non-Schmid yield behavior in single crystals. J. Mech. Phys. Solids 40, 813-833 (1992).

14. Vitek, V., Mrovec, M. \& Bassani, J. L. Influence of non-glide stresses on plastic flow: from atomistic to continuum modeling. Mater. Sci. Eng. A 365, 31-37 (2004),

15. Duesbery, M. S. On non-glide stresses and their influence on the screw dislocation core in body-centred cubic metals: I. The Peierls stress. Proc. R. Soc. Lond. A 392, 145-173 (1984).

16. Gröger, R., Racherla, V., Bassani, J. L. \& Vitek, V. Multiscale modeling of plastic deformation of molybdenum and tungsten: II. Yield criterion for single crystals based on atomistic studies of glide of $1 / 2\langle 111\rangle$ screw dislocations. Acta Mater. 56, 5412-5425 (2008).

17. Chen, Z. M., Mrovec, M. \& Gumbsch, P. Atomistic aspects of $1 / 2\langle 111\rangle$ screw dislocation behavior in $\alpha$-iron and the derivation of microscopic yield criterion. Model. Simul. Mater. Sci. Eng. 21, 055023 (2013).

18. Takeuchi, S. Core structure of a screw dislocation in the B.C.C. lattice and its relation to slip behaviour of $\alpha$-iron. Philos. Mag. A 39, 661-671 (1979).

19. Ventelon, L., Willaime, F., Clouet, E. \& Rodney, D. Ab initio investigation of the Peierls potential of screw dislocations in bcc Fe and W. Acta Mater. 61, 3973-3985 (2013).

20. Dezerald, L. et al. Ab initio modeling of the two-dimensional energy landscape of screw dislocations in bcc transition metals. Phys. Rev. B 89, 024104-024113 (2014).

21. Rodney, D. \& Proville, L. Stress-dependent Peierls potential: influence on kink-pair activation. Phys. Rev. B 79, 094108-094109 (2009).

22. Gordon, P. A., Neeraj, T. \& Mendelev, M. I. Screw dislocation mobility in BCC metals: a refined potential description for $\alpha$-Fe. Philos. Mag. Lett. 91, 3931-3945 (2010).

23. Mendelev, M. I. et al. Development of new interatomic potentials appropriate for crystalline and liquid iron. Philos. Mag. 83, 3977-3994 (2003).

24. Edagawa, K., Suzuki, T. \& Takeuchi, S. Motion of a screw dislocation in a two-dimensional Peierls potential. Phys. Rev. B 55, 6180-6187 (1997).

25. Edagawa, K., Suzuki, T. \& Takeuchi, S. Plastic anisotropy in B.C.C. transition metals. Mater. Sci. Eng. A 234-236, 1103-1105 (1997).

26. Gröger, R. \& Vitek, V. Multiscale modeling of plastic deformation of molybdenum and tungsten: III. Effects of temperature and plastic strain rate. Acta Mater. 56, 5426-5439 (2008).

27. Gröger, R. \& Vitek, V. Determination of positions and curved transition pathways of screw dislocations in bcc crystals from atomic displacements. Mater. Sci. Eng. A 643, 203-210 (2015).

28. Itakura, M., Kaburaki, H. \& Yamaguchi, M. First-principles study on the mobility of screw dislocations in bcc iron. Acta Mater. 60, 3698-3710 (2012).

29. Duesbery, M. S. \& Basinski, Z. S. The flow stress of potassium. Acta Metall. Mater. 41, 643-647 (1993).

30. Gröger, R. \& Vitek, V. Explanation of the discrepancy between the measured and atomistically calculated yield stresses in body-centred cubic metals. Phil. Mag. Lett. 87, 113-120 (2007).

31. Proville, L., Rodney, D. \& Marinica, M. C. Quantum effect on thermally activated glide of dislocations. Nat. Mater. 11, 845-849 (2012). 
32. Barvinschi, B., Proville, L. \& Rodney, D. Quantum Peierls stress of straight and kinked dislocations and effect of non-glide stresses. Model. Simul. Mater. Sci. Eng. 22, 025006-025014 (2014).

33. Gröger, R. Which stresses affect the glide of screw dislocations in bcc metals? Phil. Mag. 94, 2021-2030 (2014).

34. Srivastava, K., Gröger, R., Weygand, D. \& Gumbsch, P. Dislocation motion in tungsten: atomistic input to discrete dislocation simulations. Int. J. Plast. 47, 126-142 (2013).

35. Kocks, U., Argon, A. \& Ashby, F. Thermodynamics and Kinetics of Slip. No. 19 in International Series of Monographs in Natural Philosophy (Pergamon Press 1975).

36. Proville, L., Ventelon, L. \& Rodney, D. Prediction of the kink-pair formation enthalpy on screw dislocations in $\alpha$-iron by a line tension model parametrized on empirical potentials and first-principles calculations. Phys. Rev. B 87, 144106-144108 (2013).

37. Dezerald, L., Proville, L., Ventelon, L., Rodney, D. \& Willaime, F. Firstprinciples predictions of the kink-pair formation enthalpy on screw dislocations in bcc crystals: V, Nb, Ta, Mo, W and Fe. Phys. Rev. B 91, 094105-094107 (2015).

38. Hale, L. M., Lim, H., Zimmerman, J. A., Battaile, C. C. \& Weinberger, C. R. Insights on activation enthalpy for non-Schmid slip in body-centered cubic metals. Scripta Mater. 99, 89-92 (2015).

39. Giannozzi, P. et al. QUANTUM ESPRESSO: a modular and open-source software project for quantum simulations of materials. J. Phys: Condensed Matter 21, 395502-395519 (2009).

40. Ventelon, L. \& Willaime, F. Core structure and Peierls potential of screw dislocations in $\alpha$-Fe from first principles: cluster versus dipole approaches. J. Computer-Aided Mater. Des. 14, 85-94 (2007).

41. Clouet, E., Ventelon, L. \& Willaime, F. Dislocation core energies and core fields from first principles. Phys. Rev. Lett. 102, 055502-055504 (2009).

42. Henkelman, G., Johannesson, G. \& Jonsson, H. Progress on Theoretical Chemistry and Physics 10, 269-302 (Kluwer Academic Publishers, Dordrecht, 2000).

43. Clouet, E. Dislocation core field I. Modeling in anisotropic linear elasticity theory. Phys. Rev. B 84, 224111-224117 (2011).

44. Chaari, N., Clouet, E. \& Rodney, D. First-principles study of secondary slip in zirconium. Phys. Rev. Lett. 112, $075504-075505$ (2014).

\section{Acknowledgements}

We acknowledge support from the GENCI-CINES computer centre under grant number 2014-096821 and the Partnership for Advanced Computing in Europe (PRACE) for awarding access within the DIMAIM project to the MareNostrum III facility, based in Spain at the Barcelona Super-computing Center (BSC). D.R. acknowledges support from the Institut Universitaire de France and LABEX iMUST (ANR-10-LABX-0064) of Université de Lyon (programme 'Investissements d'Avenir', ANR-11-IDEX-0007). This work has been carried out partially within the framework of the EUROfusion Consortium and has received funding from the Euratom research and training program 2014-2018 under grant agreement No 633053. The views and opinions expressed herein do not necessarily reflect those of the European Commission. We thank Professor Vasek Vitek for his critical reading of the manuscript and his very insightful comments.

\section{Author contributions}

D.R., L.V. and F.W. designed the research. L.D. performed the DFT calculations. E.C. produced the dislocation trajectories. L.D., D.R. and E.C. analysed the data and wrote the initial manuscript. All authors discussed the data and participated in writing the final manuscript.

\section{Additional information}

Supplementary Information accompanies this paper at http://www.nature.com/ naturecommunications

Competing financial interests: The authors declare no competing financial interests

Reprints and permission information is available online at http://npg.nature.com/ reprintsandpermissions/

How to cite this article: Dezerald, L. et al. Plastic anisotropy and dislocation trajectory in BCC metals. Nat. Commun. 7:11695 doi: 10.1038/ncomms11695 (2016).

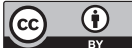

This work is licensed under a Creative Commons Attribution 4.0 International License. The images or other third party material in this article are included in the article's Creative Commons license, unless indicated otherwise in the credit line; if the material is not included under the Creative Commons license, users will need to obtain permission from the license holder to reproduce the material. To view a copy of this license, visit http://creativecommons.org/licenses/by/4.0/ 